\title{
HBD-3 regulation of the immune response and the LPS/TLR4-mediated signaling pathway
}

\author{
CHEN ZHU, NI-RONG BAO, SHUO CHEN and JIAN-NING ZHAO \\ Department of Orthopaedic Surgery, Jinling Hospital, Nanjing University School of Medicine, \\ Nanjing, Jiangsu 210002, P.R. China
}

Received December 30, 2015; Accepted August 3, 2016

DOI: $10.3892 /$ etm.2016.3579

\begin{abstract}
The aim of the study was to investigate the mechanisms of human $\beta$-defensin 3 (HBD-3) regulation of the immune response and the lipopolysaccharide/Toll-like receptor-4 (LPS/ TLR4)-mediated signaling pathway. A TLR4 extracellular gene fragment was cloned into the pET32a plasmid to determine its expression in Escherichia coli (E. coli) and purification. A dialysis labeling method was used to stain HBD-3 with fluorescein isothiocyanate (FITC). FITC-HBD-3 was used to induce the differentiation of human peripheral blood mononuclear cells (MNC) into immature dendritic cells (imDC) in vitro. Binding reactions were established using FITC-HBD-3 and sTLR4 into cell suspensions. Flow cytometry (FCM) was used to analyze the results. Western blot analysis confirmed the identity of nuclear factor- $\mathrm{\kappa B}(\mathrm{NF}-\mathrm{\kappa B})$ and was used to quantify its nuclear translocation. The results showed that, HBD-3 bound to imDC in a $\mathrm{Ca}^{2+}$-dependent manner, and sTLR4 and LPS competitively inhibited the binding. HBD-3 competitively blocked the binding of LPS and imDC by binding to imDC. HBD-3 significantly decreased the translocation of LPS-induced NF- $\mathrm{KB}$ into the nucleus. In conclusion, HBD-3 can competitively inhibit the binding of LPS and imDC through its binding to TLR4 molecules, which are expressed in imDC, thereby preventing LPS from inducing the maturity of the imDCs.
\end{abstract}

\section{Introduction}

The treatment of implant-related infections has been a concern for orthopedists for a long period of time (1). The new antibacterial agent, an endogenous antibiotic peptide known as human $\beta$-defensin-3 (HBD-3), is important in the inhibition of biofilm formation and immune regulation (2). In a previous study, it

Correspondence to: Dr Jian-Ning Zhao, Department of Orthopaedic Surgery, Jinling Hospital, Nanjing University School of Medicine, 305 East Zhongshan Road, Nanjing, Jiangsu 210002, P.R. China

E-mail: zhaojianning.0207@163.com

Key words: human $\beta$-defensin 3, Toll-like receptor-4, immature dendritic cells, nuclear factor- $\kappa \mathrm{B}$ was found that the effect of HBD-3 in killing drug-resistant Staphylococcus was significantly enhanced inside biomembranes (3). Additionally, HBD-3 inhibited the expression of the film-forming gene icaAD, and of the drug-resistant gene mecA (4). The supernatant of Staphylococcus aureus can induce stimulated osteoblasts of rats to secrete a structural and functional congener of HBD-3, the murine $\beta$-defensin 14 (MBD-14) through activation of the p38 mitogen-activated protein kinase (MAPK) (5). Furthermore, in acute suppurative osteomyelitis models infected with drug-resistant Staphylococcus aureus, the application of a p38 MAPK agonist promoted the increased release of MBD-14 in the infection site and inhibited osteomyelitis (6). These results strongly suggested that HBD-3 is useful in the prevention and treatment of implant-related infections in orthopedics.

However, the mechanisms that lead HBD-3 to induce and regulate immune responses and to inhibit bone infections remain unknown. In the present study, the effects of HBD-3 on the lipopolysaccharide/Toll-like receptor-4 (LPS/TLR4)mediated signaling pathway and on the maturation process of dendritic cells were determined and analyzed to clarify the mechanisms of action of the antimicrobial peptide.

\section{Materials and methods}

Soluble expression, purification and identification of TLR4 extracellular segments in Escherichia coli (E. coli). Plasmid was constructed to determine the protein expression. The pCDNA3-TLR4 plasmid was purified from E. coli using a plasmid mini kit (Hangzhou Vitagene Biochemical, Hangzhou, China) according to the manufacturer's instructions. Polymerase chain reaction (PCR) was used to amplify a fragment from the purified plasmid that contains a human TLR4 template, using the forward primer, 5'-CGGGATCCATGATGTCTGCC-3' (containing a BamHI restriction site) and reverse primer, 5'-GCAAGCTTCTTATTCATCTGACAGG-3' (containing a HindIII restriction site) (Shanghai Boya Biotechnology Co., Ltd., Beijing, China). The PCR conditions used were: pre-denaturation at $94^{\circ} \mathrm{C}$ for $4 \mathrm{~min}, 30$ cycles of denaturation at $94^{\circ} \mathrm{C}$ for $40 \mathrm{sec}$, followed by annealing at $53^{\circ} \mathrm{C}$ for $50 \mathrm{sec}$, and extension at $72^{\circ} \mathrm{C}$ for $1 \mathrm{~min}$, and a final extension at $72^{\circ} \mathrm{C}$ for $10 \mathrm{~min}$. PCR products were extracted according to the instructions of the 'DNA rapid purification/recycling kit', and the purified products were preserved at $-20^{\circ} \mathrm{C}$. 
PCR products and pET32a were subjected to a doubleenzyme digestion by BamHI and HindIII. After extraction of the appropriate fragments, a ligation was set up using T4 DNA ligase. Ligations were used to transform E.coli. and were placed onto LB medium containing ampicillin. After plasmid purification from single colonies and enzyme digestion to verify the restriction pattern of the constructs, sequencing was used to select the right recombinant expression vector pET32a-TLR4, containing the TLR4 segment fused to a thioredoxin tag. BL21 (DE3) plysS competence bacteria were transformed with the purified vector and selected in LB medium with ampicillin following incubation at $37^{\circ} \mathrm{C}$ overnight. For the expression, a new culture was created into the same $1 \%$ of the total type of medium using starter culture, at $37^{\circ} \mathrm{C}$ in an oscillating incubator. When the optical density reached $\mathrm{A}_{600} \mathrm{~nm}=0.6-1.0$, IPTG was added to a final concentration of $1 \mathrm{mmol} / \mathrm{l}$ and the culture was induced for an additional 4 hours. The induced cultures were centrifuged, the pellets were resuspended into buffer solution $\mathrm{A}(10 \mathrm{mmol} / \mathrm{l}$ imidazole, $300 \mathrm{mmol} / \mathrm{l} \mathrm{NaCl}$ and $50 \mathrm{mmol} / 1 \mathrm{NaH}_{2} \mathrm{PO}_{4}, \mathrm{pH} 8.0$ ), ultrasonication was performed and the resulting suspensions were then centrifuged at $3,000 \mathrm{x} \mathrm{g}$ for $10 \mathrm{~min}$ at $4^{\circ} \mathrm{C}$. The supernatants containing the soluble protein fractions were saved.

Protein purification. Soluble protein fractions were allowed to pass through an $\mathrm{Ni}^{2+}$-NTA column (Qiagen, Valencia, CA, USA) that was previously balanced with buffer solution A by gravity flow. Buffer solution B $(20 \mathrm{mmol} / \mathrm{l}$ imidazole, $300 \mathrm{mmol} / \mathrm{l} \mathrm{NaCl}$ and $50 \mathrm{mmol} / 1 \mathrm{NaH}_{2} \mathrm{PO}_{4}, \mathrm{pH} 8.0$ ) was then used to rinse the column. The target protein was eluted from the column with buffer solution $\mathrm{C}(250 \mathrm{mmol} / \mathrm{l}$ imidazole, $300 \mathrm{mmol} / \mathrm{l} \mathrm{NaCl}$ and $50 \mathrm{mmol} / 1 \mathrm{NaH}_{2} \mathrm{PO}_{4}, \mathrm{pH}$ 8.0). Subsequently, the target protein was ultrafiltrated and concentrated, and fully dialyzed in $50 \mathrm{mmol} / \mathrm{l}$ phosphate-buffered saline (PBS) (pH 7.2) to clear away the imidazole and salt contents. The final product was the purified TLR4-Trx fusion protein.

Identification of TLR4-Trx protein using western blot analysis. Purified TLR4-Trx protein $(300 \mu \mathrm{g})$ was separated with sodium dodecyl sulfate-polyacrylamide gel electrophoresis (SDS-PAGE), and transferred to a nitrocellulose filter. The filter was blocked with $30 \mathrm{~g} / 1$ dried skimmed milk at $4^{\circ} \mathrm{C}$ overnight. The following morning, HRP-anti His antibody (rabbit anti-mouse monoclonal antibody, catalog no. 201321) was diluted in blocking buffer (1:1,000; Nanjing Jingmei Chemical Co., Ltd., Nanjing, China) and incubated with the nitrocellulose membrane at $37^{\circ} \mathrm{C}$ for $2 \mathrm{~h}$. TBST was used to wash the membrane 5 times for $10 \mathrm{~min}$; and color was developed using a DAB color development kit for the appropriate amount of time. The PVDF membrane was dipped into distilled liquid to terminate color development and air-dried. Images were captured to confirm the size of the target protein. The protein fraction from BL21 (DE3)/PET32a was used as a negative control.

Preliminary study of the regulation mechanism of $H B D-3$ on dendritic cells. HBD-3 was diluted to $1 \%$ in $0.025 \mathrm{~mol} / 1$ carbonate buffer at $\mathrm{pH}$ 9.0, and placed into a dialysis bag. Fluorescein isothiocyanate (FITC) was prepared as a $0.1 \mathrm{mg} / \mathrm{ml}$ solution in the carbonate buffer solution, and used as a diluent in a $10 \mathrm{mg} / \mathrm{ml}$ globulin solution. The dialysis bag was immersed into the globulin FITC solution containing 10 -fold its volume. Dialysis occurred at $4^{\circ} \mathrm{C}$ on a stirring plate with a magnet for $2 \mathrm{~h}$. The dyalized contents of the bag were immediately filtered using a Sephadex G50 gel to remove the free fluorescein and aliquots were packed and stored at $4^{\circ} \mathrm{C}$. The resulting F/P ratio of the stained FITC-HBD-3 was 2.

In vitro immature dendritic cell (imDC) induction. Peripheral blood from healthy adult volunteers was processed with heparin as an anticoagulation agent. Mononuclear cells (MNCs) were isolated via density gradient centrifugation (Ficoll). PBS was used to wash away platelets, MNCs were allowed to adhere to the vessel wall for $3 \mathrm{~h}$ at $37^{\circ} \mathrm{C}$ and the supernatant was discarded. The remaining non-adherent cells were gently rinsed with pre-warmed $\left(37^{\circ} \mathrm{C}\right.$ ) RPMI-1640 (Gibco, Grand Island, NY, USA). Adherent MNCs were scraped off the wall, and suspended in a $1 \times 10^{9}$ cells/1 solution in RPMI-1640 supplemented with $100 \mathrm{ml} / \mathrm{l}$ new-born calf serum (Hangzhou Sijiqing Biological Engineering Material Co., Ltd., Hangzhou, China). Susequently, $100 \mu \mathrm{g} / \mathrm{l} \mathrm{rhGM}-\mathrm{CSF}$ and $100 \mu \mathrm{g} / \mathrm{l} \mathrm{hhIL-4}$ (both from Peprotech, Inc., Rocky Hill, NJ, USA) were added into the MNC suspension and the cells were cultured in an incubator at $37^{\circ} \mathrm{C}$. Half the amount of the medium was exchanged every 3 days and supplementing cytokines were added when necessary. Cell morphology was observed under a confocal microscope. After seven days, cells were collected, the phenotype and function of imDC were analyzed, and the supernatant of each group collected for measuring the cytokines present in the supernatant.

Cell binding tests. HBD-3 was bound to imDC. Briefly, imDCs were suspended in buffer solution $\mathrm{A}\left(5 \mathrm{mmol} / \mathrm{C} \mathrm{Ca}^{2+}\right)$ and the cell density was adjusted to $5 \times 10^{9} / 1$. FITC-HBD-3 was then added to $200 \mu 1$ of cell suspension to a final concentration of $15 \mathrm{mg} / \mathrm{l}$, and the reaction was allowed to proceed for $30 \mathrm{~min}$ at $37^{\circ} \mathrm{C}$ in the dark. The potential inhibitor sTLR4 was added to another group of cells to a final concentration of $30 \mathrm{mg} / \mathrm{l}$, and FITC-HBD-3 was added $10 \mathrm{~min}$ later. The reaction was allowed to proceed for $30 \mathrm{~min}$ at $37^{\circ} \mathrm{C}$ in the dark. The cells were then washed 3 times using PBS prior to flow cytometry (FCM). Unlabelled FITC-HBD-3 was used as a negative control.

To identify HBD-3 as a competitive inhibitor of the binding between LPS and imDC, imDCs were resuspended in buffer solution A to an adjusted cell density of $5 \times 10^{9} / 1$. HBD-3 (15 mg/l) was added into $200 \mu \mathrm{l}$ cell suspension for the reaction. A group of cells with no added HBD-3 served as a negative control. The specificity of HBD-3 was assessed using anti-HBD-3 pAb. The reaction was allowed to proceed for $30 \mathrm{~min}$ at $37^{\circ} \mathrm{C}$ in the dark, and then PBS was used to wash the cells 3 times prior to FCM analysis.

Western blot analysis. Western blot analysis of the binding of HBD-3 and sTLR4 was performed. Briefly, $2 \mu \mathrm{g}$ sTLR4 extracellular proteins were processed by 2-mercaptoethanol loading buffer. After separation, a 13\% SDS-PAGE was stained by CBB R250. The proteins were then transferred to a PVDF membrane at $60 \mathrm{~V}$ for $3 \mathrm{~h}$ under constant pressure. The PVDF membrane was immersed into buffer solution B for $2 \mathrm{~h}$, followed by buffer solution B containing $5 \mu \mathrm{g} / \mathrm{ml} \mathrm{HBD}-3$, for $3 \mathrm{~h}$ at room temperature. The membrane was washed 5 times 
(1:2,000). Mouse anti-human HBD-3 mAb HYP131-11 (R\&D Systems, Inc., Minneapolis, MN, USA) was added to the washed membrane, and the binding was allowed to proceed for $60 \mathrm{~min}$ at room temperature. The 1:2,000 sheep anti-rat IgG (Sigma, St. Louis, MO, USA) was incubated with the membrane for $60 \mathrm{~min}$ at room temperature. The membrane was then washed and color was developed using a DAB color development kit prior to capturing the image.

Nuclear proteins were extracted strictly in accordance with the instruction of a kit (Pierce Biotechnology, Inc., Rockford, IL, USA) and the concentration of the extraction was measured using the Bradford method. The activity of nuclear factor- $\kappa \mathrm{B}(\mathrm{NF}-\kappa \mathrm{B})$ was detected using western blot analysis. Nuclear protein extracts $(5 \mu \mathrm{g})$ were separated with $12 \%$ SDS-PAGE. The proteins were then transferred to a PVDF membrane for $3 \mathrm{~h}$. The transferred membrane was blocked in PBS-T that contained 3\% dried skimmed milk for $2 \mathrm{~h}$. Subsequently, 1:1,500 HRP-sheep anti-rat IgG was added and the solution was incubated with the membrane for $60 \mathrm{~min}$ at room temperature. The membrane was then washed as described above, color was developed using a DAB color kit and images were captured.

Statistical analysis. SPSS 19.0 software was used for statistical analysis. Quantitative data were presented as mean \pm standard deviation. The Student's t-test was used in group comparisons. Enumeration data were presented as a percentage (\%) and the $\chi^{2}$ test was applied in group comparisons. $\mathrm{P}<0.05$ was considered statistically significant.

\section{Results}

Soluble expression of TLR4 extracellular fragment in E. coli, purification and identification. A single amplicon of the expected size (1,911 bp) was detected after gel electrophoresis and ethidium bromide staining (Fig. 1). Restriction analysis of vector pET32a-TLR with HindIII and BamHI enzyme digestions showed the expected pattern $(5,900 \mathrm{bp}$ for the vector fragment and 1,911 bp for the amplicon) (Fig. 2). Sequencing results confirmed the correct exogenous DNA insert pET32a-TLR.

Binding of HBD-3 and imDC. FCM analysis showed that HBD-3 can directly bind to imDC in $5 \mathrm{mmol} / \mathrm{l}$ binding buffer (Fig. 3). Western blot analysis showed that HBD-3 can bind to sTLR4 protein (Fig. 4).

Competitive inhibition tests. sTLR4 extracellular protein can partially inhibit the binding of HBD-3 and imDC, indicating that the binding site of HBD-3 and imDC is associated with TLR4 (Fig. 5). LPS can competitively inhibit the binding of HBD-3 and imDC, indicating that HBD-3 can bind TLR4, which is found on the surface of imDC (Fig. 6). After the addition of anti-HBD-3 pAb, the inhibitory effect of HBD-3 disappeared, indicating that the inhibition of HBD-3 had specificity (Fig. 7). The western blot analysis revealed that LPS stimulation can significantly increase the nuclear translocation of NF- $\mathrm{KB}$, which is the nuclear transcription factor of imDCs, although the translocation was significantly decreased by high concentrations of HBD-3. After, the addition of anti-HBD-3

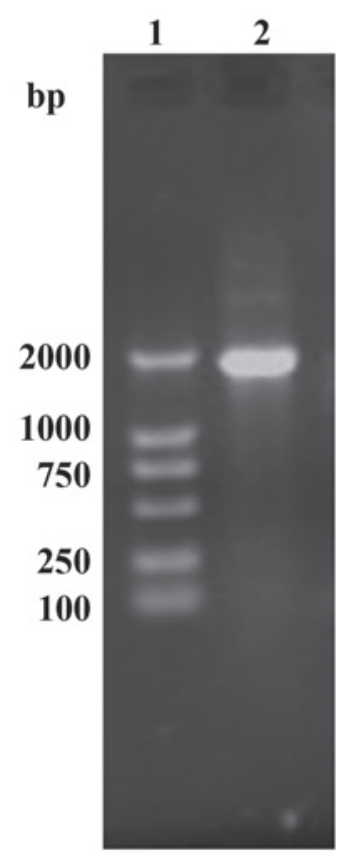

Figure 1. PCR amplified TLR4 extracellular fragment gene segment. PCR, polymerase chain reaction; TLR4, Toll-like receptor-4.

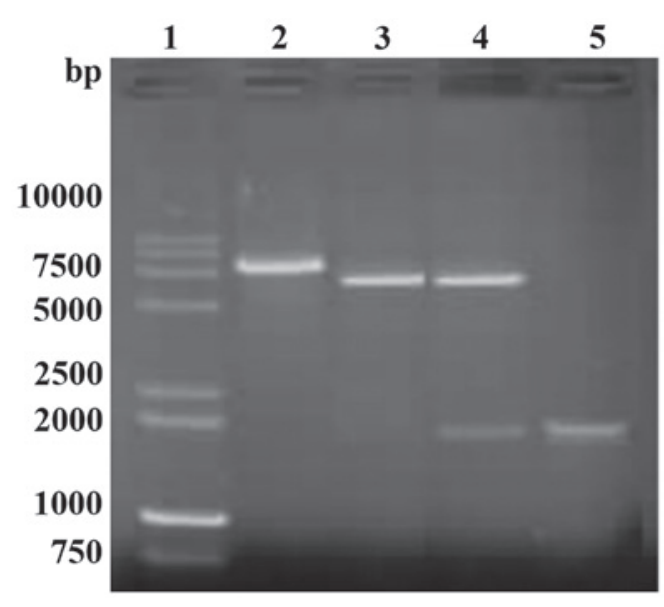

Figure 2. Restriction analysis of recombinant plasmid pET32a-TLR4 TLR4, Toll-like receptor-4.

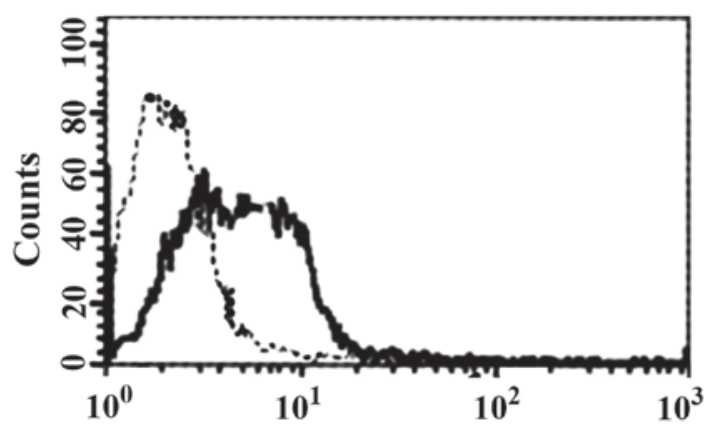

Figure 3. Binding of HBD-3 and imDC. HBD-3, human $\beta$-defensin 3; imDC, immature dendritic cells.

$\mathrm{pAb}$, the inhibitory effect of HBD-3 disappeared, indicating that the inhibition of HBD-3 is specific (Fig. 8). 


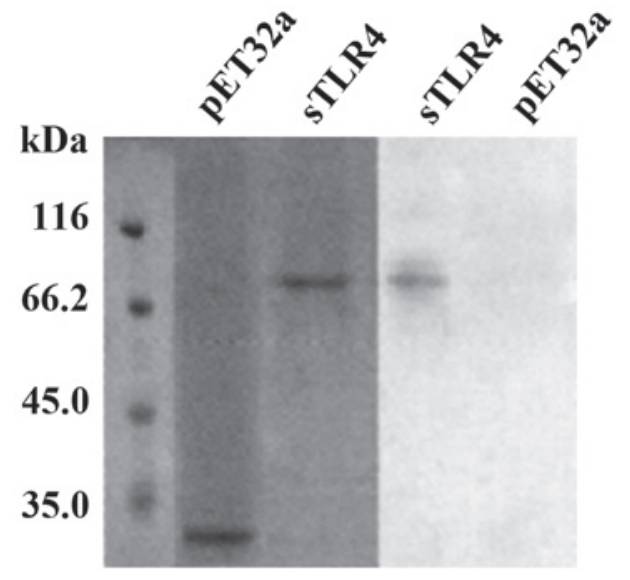

Figure 4. Binding of HBD-3 and TLR4. HBD-3, human $\beta$-defensin 3; TLR4, Toll-like receptor-4.

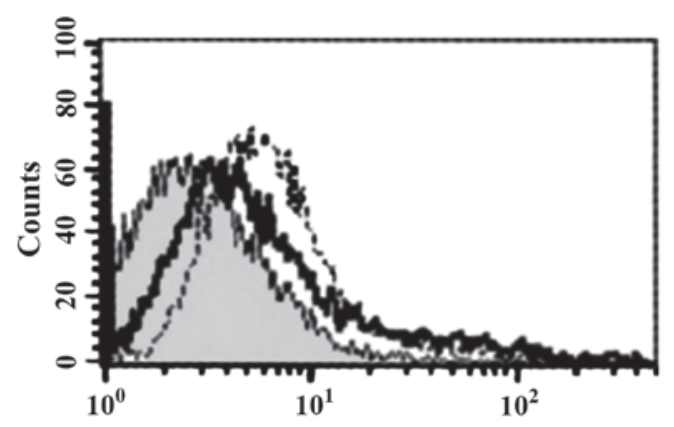

Figure 5. Competitive inhibition test of HBD-3. HBD-3, human $\beta$-defensin 3.

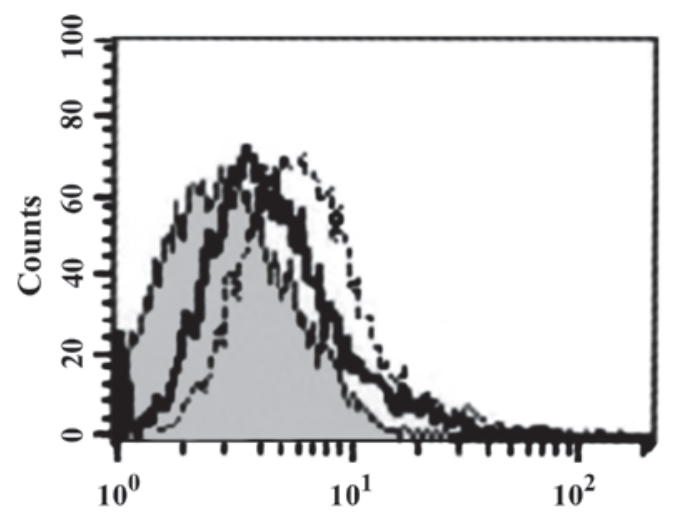

Figure 6. Competitive inhibition test of LPS. LPS, lipopolysaccharide.

\section{Discussion}

Previous findings revealed that bacterial infection of osteoblasts can stimulate the secretion of cytokines, chemokines, and endogenous antimicrobial peptide $\beta$-defensin (7). Thus, osteoblasts regulate bone metabolism and play a role in the regulation of an active immune defense response and acquired immunity. $\beta$-defensin, a new type of bacterial infection inhibitor characterized by its wide antibiotic spectrum, rapid action, low drug resistance rate, innate immune compatibility and its unique role in the immune regulation of bone infection, has drawn attention in clinical and basic research settings (8).

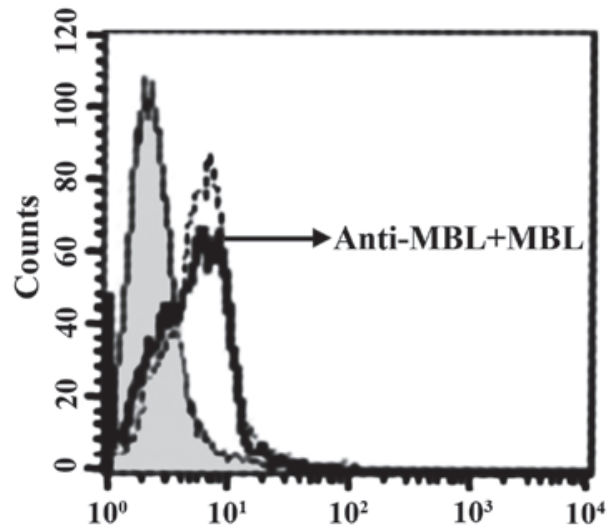

Figure 7. Inhibition specificity of HBD-3. HBD-3, human $\beta$-defensin 3.

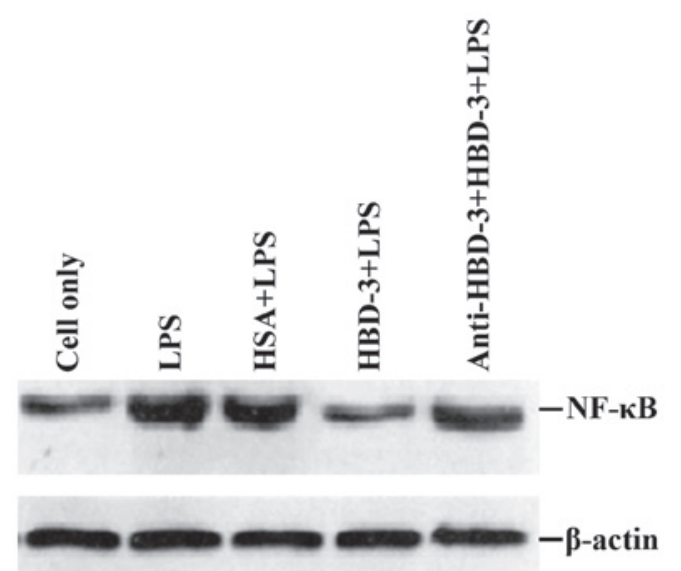

Figure 8. Western blot analysis of the relative activity of NF- $\kappa$ B. NF- $\kappa B$, nuclear factor- $\kappa B$.

Shi et al (9) demonstrated that Staphylococcus aureusor Candida albicans-contaminating maxillofacial silicone elastomers were significantly inhibited or killed by immersing them into an HBD-3 solution for only $30 \mathrm{~min}$. Other authors found that Staphylococcus aureus supernatants can induce the release and expression of HBD-3 through TLR2 and TRL4 on the surface of osteoblasts (10). Furthermore, the expression of HBD-3 in bone tissues of acute infection sites and on the boundary tissues of periprosthetic joint infections was doubled, indicating that endogenous antimicrobial peptide HBD-3 is a first defense line after bacterial invasion into bone tissues. In vitro HBD-3 inhibits TLR4-mediated myeloid differentiation factor 88 (MyD88) and TIR structural domain adaptor molecule (TRIF) pathways, and inhibits the activation of NF- $\kappa \mathrm{B}$ (11). In vitro and in vivo HBD-3 has been shown to inhibit the LPS stimulation of macrophages to secrete tumor necrosis factor- $\alpha$ (TNF- $\alpha$ ) and interleukin-6 (IL-6). Therefore, it was postulated that HBD-3 is, not only an inflammatory chemokine, but also a suppressant immunomodulator, with balancing and bidirectional adjusting effects in antisepsis and anti-inflammation. Another study confirmed that in an immune reaction, the structural and functional congener of HBD-3, MBD-14, initially confronts a microbial invasion through the innate immune response and then promotes the extensive chemotaxis of MNC (Mo), macrophages (MФ), neutrophils, 
imDC and T lymphocytes in a chemokine receptor 6 (CCR6)and CCR2 receptor-dependent manner (12). HBD-3 can also activate the MAPK extracellular signal-regulated kinase (ERK) signaling pathway, M $\Phi$ and antigen-presenting cells (APCs) through NF-kB-dependent TLR-1 and TLR-2 receptor pathways $(13,14)$.

TLR is an important pattern recognition receptor in the natural immune system, which can, not only mediate the identification of pathogenic microorganisms and their products, but also participate in the acquired immune response. It has been recognized as a bridge that binds natural immunity with acquired immunity (15). TLR is extensively expressed in imDC, $\mathrm{M} \Phi$, and Mo, and plays an important role in identifying gram-positive teichoic acid, heat shock protein 60 , LPS and the signaling pathways mediated by LPS (16). Based on those and our own findings, we conclude that HBD-3 binds to imDC in a $\mathrm{Ca}^{2+}$-dependent manner, and that sTLR4 and LPS can competitively inhibit binding. HBD-3 can competitively inhibit the binding of LPS and imDC by binding with imDC. HBD-3 significantly inhibits the translocation of LPS-induced $\mathrm{NF}-\kappa \mathrm{B}$ into the nucleus. Thus, HBD-3 can competitively inhibit the binding of LPS and imDC by binding with TLR4 molecules expressed in imDCs, thereby preventing LPS from inducing the maturity of imDC.

\section{Acknowledgements}

The present study was supported by the National Natural Science Foundation of China (grant no. 81401815), the China Postdoctoral Science Foundation (grant no. 2015M582900) and the Jiangsu Postdoctoral Science Foundation (grant no. $1501146 \mathrm{C})$.

\section{References}

1. Montanaro L, Speziale P, Campoccia D, Ravaioli S, Cangini I, Pietrocola G, Giannini S and Arciola CR: Scenery of Staphylococcus implant infections in orthopedics. Future Microbiol 6: 1329-1349, 2011.

2. Zhu C, Tan H, Cheng T, Shen H, Shao J, Guo Y, Shi S and Zhang X: Human $\beta$-defensin 3 inhibits antibiotic-resistant Staphylococcus biofilm formation. J Surg Res 183: 204-213, 2013.

3. Ning R, Zhang X, Guo X and Li Q: Staphylococcus aureus regulates secretion of interleukin- 6 and monocyte chemoattractant protein-1 through activation of nuclear factor kappaB signaling pathway in human osteoblasts. Braz J Infect Dis 15: 189-194, 2011.
4. Jarczak J, Kościuczuk EM, Lisowski P, Strzałkowska N, Jóźwik A, Horbańczuk J, Krzyżewski J, Zwierzchowski L and Bagnicka E: Defensins: natural component of human innate immunity. Hum Immunol 74: 1069-1079, 2013.

5. Zhu C, He N, Cheng T, Tan H, Guo Y, Chen D, Cheng M, Yang $\mathrm{Z}$ and Zhang $\mathrm{X}$ : Ultrasound-targeted microbubble destruction enhances human $\beta$-defensin 3 activity against antibiotic-resistant Staphylococcus biofilms. Inflammation 36: 983-996, 2013.

6. Hinrichsen K, Podschun R, Schubert S, Schröder JM, Harder J and Proksch E: Mouse beta-defensin-14, an antimicrobial ortholog of human beta-defensin-3. Antimicrob Agents Chemother 52: 1876-1879, 2008.

7. Röhrl J, Yang D, Oppenheim JJ and Hehlgans T: Identification and biological characterization of mouse beta-defensin 14 , the orthologue of human beta-defensin 3. J Biol Chem 283: 5414-5419, 2008 .

8. Hancock RE and Sahl HG: Antimicrobial and host-defense peptides as new anti-infective therapeutic strategies. Nat Biotechnol 24: 1551-1557, 2006.

9. Shi Y, Song W, Feng ZH, Zhao YT, Li F, Tian Y and Zhao YM: Disinfection of maxillofacial silicone elastomer using a novel antimicrobial agent: recombinant human beta-defensin-3. Eur J Clin Microbiol Infect Dis 28: 415-420, 2009.

10. Varoga D, Wruck CJ, Tohidnezhad M, Brandenburg L, Paulsen F, Mentlein R, Seekamp A, Besch L and Pufe T: Osteoblasts participate in the innate immunity of the bone by producing human beta defensin-3. Histochem Cell Biol 131: 207-218, 2009.

11. Semple F, MacPherson H, Webb S, Cox SL, Mallin LJ, Tyrrell C, Grimes GR, Semple CA, Nix MA, Millhauser GL, et al: Human $\beta$-defensin 3 affects the activity of pro-inflammatory pathways associated with MyD88 and TRIF. Eur J Immunol 41: 3291-3300, 2011.

12. Röhrl J, Yang D, Oppenheim JJ and Hehlgans T: Human beta-defensin 2 and 3 and their mouse orthologs induce chemotaxis through interaction with CCR2. J Immunol 184: 6688-6694, 2010

13. Zhu C, Qin H, Cheng T, Tan HL, Guo YY, Shi SF, Chen DS and Zhang XL: Staphylococcus aureus supernatant induces the release of mouse $\beta$-defensin-14 from osteoblasts via the p38 MAPK and NF-кB pathways. Int J Mol Med 31: 1484-1494, 2013.

14. Zhu C, Wang J, Cheng T, Li Q, Shen H, Qin H, Cheng M and Zhang X: The potential role of increasing the release of mouse $\beta$-defensin-14 in the treatment of osteomyelitis in mice: a primary study. PLoS One 9: e86874, 2014.

15. Wang M, Chen Y, Zhang Y, Zhang L, Lu X and Chen Z: Mannan-binding lectin directly interacts with Toll-like receptor 4 and suppresses lipopolysaccharide-induced inflammatory cytokine secretion from THP-1 cells. Cell Mol Immunol 8: 265-275, 2011.

16. Spadaro M, Montone M, Arigoni M, Cantarella D, Forni G, Pericle F, Pascolo S, Calogero RA and Cavallo F: Recombinant human lactoferrin induces human and mouse dendritic cell maturation via Toll-like receptors 2 and 4. FASEB J 28: 416-429, 2014. 